\title{
Optimal Design and Modeling of Sustainable Buildings Based on Multivariate Fuzzy Logic
}

\author{
Ridhima Mehta \\ School of Computer and Systems Sciences, Jawaharlal Nehru University, New Delhi 110067, India
}

Corresponding Author Email: ridhim75_scs@jnu.ac.in

https://doi.org/10.18280/ijsdp.160120

Received: 6 November 2020

Accepted: 6 February 2021

\section{Keywords: \\ energy efficiency, environment quality, fuzzy logic, membership function,} sustainable building

\begin{abstract}
The exemplary design of green buildings incorporates multiple correlated parameters that should be optimally selected and updated to ensure effective energy management and sustainable impact of building architecture on the ecosystem. In this paper, the adaptive modeling and development of such sustainable buildings with several unpredictable and qualitative attributes is implemented with multiple-input multiple-output (MIMO) fuzzy control system. The outputs of the proposed fuzzy corroborated sustainable building paradigm comprise of specific characteristics employed for assessing the optimal performance, viz. energy efficiency, user satisfaction, resources optimization, and environment quality. These diverse system criteria measured using the proposed fuzzy optimization model are plotted against their actual theoretical values for each data case. Furthermore, the sample dataset for the proposed sustainable building model is validated through simulation results in terms of mean absolute error and logarithmic quotient error in the estimation of various fuzzy output variables evolving with the dataset size.
\end{abstract}

\section{INTRODUCTION}

Recently, the concept of green buildings approach has gained massive evolution on account of the beneficial impacts posed by these sustainable structures. A green building is one that is optimally designed with minimal operational cost and exploitation of energy harvesting techniques to derive the large-scale ambient energy from the natural environment. The significant advantages offered by this sustainable development perception modeling include energy conservation, lower water consumption, recycling of construction materials, waste minimization, preservation of natural resources, etc. The sustainable construction and maintenance of buildings is aimed at meeting the needs of the present without compromising the potential of future generations.

Environment conducive construction and operation practices are typically deployed in the design of the green performance-efficient buildings. Moreover, from the perspective of user communities residing in these buildings, user satisfaction issues regarding the health and comfort of residents is also a consequential concern. Economical green system design techniques have currently drawn considerable attention to address the key challenges for the sustainable development of green buildings. Nevertheless, with global rising awareness for the climate change due to emission of harmful greenhouse gases from conventional energy sources, the presented energy harvesting system using the naturally replenished ambient energy as green sustainable energy sources is appealing as a relatively novel and promising solution. The green buildings framework aims to achieve the energy-proficient solutions for globally efficient systems from the environmental impacts and the quality-of-experience (QoE) prospectives of end-users. The recent emphasis is on green systems design and management to contribute significantly to reducing the global warming effects by minimizing the demand on non-renewable resources.

The proposed ecologically-feasible model relies on environmental friendly techniques to generate energy from the renewable resources that do not cause carbon dioxide emissions, e.g., solar cells, wind turbines, electromagnetic waves, thermal energy, etc. This can subsequently alleviate the energy costs and potentially hazardous effects of harmful gas emissions caused on the environment. In addition, the energy should be accumulated in such a way that the stored energy can be effectively used under the conditions that there is always energy available when needed. The green setup paradigm for the sustainable development of effectively automated smart buildings can essentially facilitate mitigation of the greenhouse effect and reduced operational expenditure. The optimized sustainable building architecture pertaining to energy limitations and environmental considerations is aimed at minimizing the global warming impact by reducing the radiations caused by greenhouse gas emissions. These gases usually generated in various industrial applications can be potentially mitigated through the ascending dependence on renewable energy sources as the emerging green sources of clean energy.

Multi-dimensional decision-making framework pursued with the flexible fuzzy logic concept has recently attracted considerable research interest. Green building practices among the traditional construction procedures are implemented predicated on the global rising concerns of climate change and resource limitations in energy sectors, in addition to the improved quality of life requirements. In this work, we consider multiple factors affecting the sustainable development of green building design through the implementation of fuzzy logic technique. These imprecise and uncertain characteristics influencing the robust and persistent 
building structure can be optimally modeled by multi-attribute decision-making scheme based on fuzzy logic. Different input attributes contemplated in the proposed fuzzy-based sustainable building model comprise of (1) the on-site renewable energy consumption, (2) indoor environmental air quality, (3) water conservation, (4) services accessibility with public facilities, (5) reused material content employed in construction practices, and (6) greenhouse gas emissions. Moreover, the performance of these buildings is assessed through various heterogeneous criteria that serve as different fuzzy outputs. These performance parameters incorporate the energy efficiency, user satisfaction, resources optimization and environment quality factors associated with the adaptive design of sustainable infrastructure.

\subsection{Related work}

In this section, a literature review has been conducted specifying the relevant studies in the area of sustainable design architecture of performance-oriented green buildings. A multiobjective building design model is developed by Yao [1] for prospective sustainable construction and global economic growth. This model is aimed at the prediction of the operation period with improved energy efficiency and environmental performance, without consideration of the recycled material content and users' perspective criteria. Ahn et al. [2] formulated an integrated construction process to facilitate cooperation among decision-makers and stakeholders for developing resilient buildings at the early stages of sustained configuration. Abualrejal et al. [3] introduced the awareness about green building methods dealing with the energy efficiency issues. This paper employed the inductive and qualitative analysis technique to retrieve the constructive sustainability and material conservation criteria. It focused on the optimal performance of energy, without taking into account the resource efficiency and environmental protection issues. Ho and So [4] developed a self-sustainable city model for resource management and household waste reduction. This structural model applied various environment constructs for analyzing the adjusted R-squared and t-values of community based culture-constrained attributes. Singh [5] investigated the impact of various materials on the design and development of sustainable buildings which cause reduced environmental degradation and concurrently with the lower operating costs. Recycling and reuse principles are adopted together with the selection of natural green resources for achieving optimal green building objectives. Blanco et al. [6] evaluated the performance of green buildings envelope through the multiple linear regression technique. It aimed at alleviating the external surface temperature and enhancing the energy proficiency of buildings to realize environment sustainability by validation over the experimental data. The green rating systems are studied by Oberti and Plantamura [7] to account for the development of naturally feasible environment. The most effective indoor and outdoor elements are considered in the proposed mechanism in an effort to design energy conserving sustainable sites with the occupants' well-being as the primary concern. However, compared with our approach, this work [7] did not consider the recyclability and efficient water consumption techniques for enhanced sustainable performance of green building design. Patnaik et al. [8] assessed the significance of indoor environment quality factor in the design of green buildings. The impact of this parameter is computed by measuring the prevailing degrees of user satisfaction, climate profile and emissions of volatile organic compounds from the conventional building materials. As opposed to this, we effectuate the energy conservation, resource management, and client satisfaction objectives, along with the improved environment characteristics through the multiple-input multiple-output (MIMO) fuzzy design. Li et al. [9] employed the isolated forest algorithm and average value interpolation techniques to achieve the energy preservation goal in green building operations.

Recently, an extensive review of information and communications technology architecture to effectuate the concept of green buildings in existing construction enterprises is performed by Martins et al. [10]. This framework considered the emphasis of environmental stability and user-centered convenience for accomplishing the sustainable operation of various design applications. Bhatt et al. [11] employed several natural phenomena of rainwater harvesting, solar energy generation, standard illumination, etc. to facilitate the development of energy- and cost-effective buildings. Singh et al. [12] introduced the GRIHA rating system for assessing the green building schemes and administering the future sustainable construction. Various aspects of acquiring sustainability are examined with their associated rating points in this employed strategy, while mutually providing incentives for the economical growth. Borissova [13] presented combinatorial optimization model for determining the green building principles based on utility function associated with the experts' knowledge. In this model, multiple criteria affecting the modern building projects weighted by appropriate coefficients are contemplated to retrieve the aggregate group decision estimations. Rajagopal and Karuppiah [14] employed numerical analysis technique of response surface methodology for utilization of recycled waste materials in manufacturing industry. The proposed framework evaluated cost-effective and sustainable development of built up infrastructure projects with improved environment quality. Petrova et al. [15] employed data mining and semantic modeling techniques for predicting the performance of sustainable building design. This is accomplished by retrieving patterns in the large volumes of existing data utilized by the building architects. Relationship analysis among various parameters influencing the adoption of green building conventions is performed by Saleh et al. [16]. For this, they used the partial least squares approach to structural equation modeling for synchronic estimation of multiple decision variables. Contrary to these recently proposed sustainable building models existing in literature, we employ the artificial intelligence strategy of fuzzy problem-solving reasoning algorithm for the adaptive setting and assessment of multiple attributes effecting the planning, operation and maintenance of sustainable building architectures.

\subsection{Contribution}

Despite the aforementioned previous works, in this paper, we have proposed a novel fuzzy logic-based sustainable building model which examines the uncertain impact of different parameters affecting the intelligent development of green buildings. To the best of the author's knowledge, no prior work has applied the computationally intelligent fuzzy reasoning methodology for implementation of the sustainable building planning and operation. In this paper, we have scrutinized multiple performance indicators for attaining optimal design and construction practices of green buildings 
including the energy efficiency, user satisfaction, resources optimization, and environment quality. These diverse attributes typically possess disparate range of values and varying units. Therefore, the fuzzy logic system evidently appears as the potential and effective method for retrieving the multidimensional system optimization. In contrast, the earlier works have employed only one or two of these objectives for the design optimization of sustainable building. The performance of the proposed model is captured from a multidimensional scope using the fuzzy control approach. Measuring the feasibility performance of sustainable building design from the perspective of adaptive fuzzy control system which models different attributes associated with the resource efficiency and environment quality is a scarce methodology on literature review. Because of the imprecise and ambiguous data coupled with the optimal design and operation of green buildings, fuzzy reasoning mechanism apparently constitutes a convenient tool for the initial design stages of sustainable and smart buildings. The advantages of the deployed MIMO fuzzy system is the coherent and analytical modeling of the developed sustainable structure, contemplating various dimensions influencing the performance of green building design. Moreover, it facilitates the decision-making implementation that emulates the human reasoning paradigm. This multivariable Mamdani-type fuzzy logic controller determines the set of optimal output parameters assessing the performance analysis of autonomous sustainable development. The nonlinear MIMO fuzzy control process operates in a selforganizing decentralized way for exploiting the fuzzy rule base and making corresponding inferences.

The developed system model employing fuzzy decisionmaking with multiple attributes is based on two key steps. The first step is the identification of multiple qualitative parameters for evaluating the sustainability performance of environmentally optimal buildings. In this step, the notion of expert knowledge is utilized to capture the implicit uncertainties of various attributes that facilitates the decisionmaking phenomenon via the fuzzy logic concept. The key attributes influencing the efficient layout and deployment of such environment conducive buildings including renewable energy consumption, indoor air quality factor, water conservation, services accessibility, recycled material, and greenhouse gas emissions are contemplated as the input variables to the formulated fuzzy inference methodology. The presented fuzzy design model combines these heterogeneous parameters by employing the membership functions and fuzzification interfaces. In the second step, the fuzzy inference system captures the expert knowledge through the prespecified fuzzy rules database. The simultaneous impact of multiple decision criteria incorporated in the fuzzy logic controller is considered for assessment of the feasibility performance factor. The developed fuzzy inference system with several input criteria is implemented using Fuzzy Logic Toolbox in Matlab simulation tool. Besides, we evaluate the validation performance of the proposed model by estimating the system error measurements including mean absolute error and logarithmic quotient error for assessing the viability prediction of various output parameters for a particular sample dataset.

\subsection{Organization of the paper}

The remainder of this paper is organized as follows. In section 2, we introduce some elementary knowledge of the fuzzy logic concept. The proposed fuzzy logic-based sustainable buildings model is discussed in section 3. In section 4 , we provide the detailed simulation analysis and the model validation results for the developed fuzzy system. Finally, the whole work is concluded in section 5, with practicable future research directions.

\section{PRELIMINARIES OF FUZZY LOGIC THEORY}

Fuzzy logic has emerged as a potential computation intelligence mechanism to represent the multiple-criterion decision-making framework. Perceived as a generalization of the deterministic logic, this technique commenced by Zadeh [17] has been widely studied and applied for mathematical modeling of multivariate logic design with imprecise human reasoning and experiences. Several parameters with diverse units, dynamic range of continuous values and implicit uncertainties can be expressed through the fuzzy logic process. Based on the notion of non-binary intermediate logic, the linguistic constructs such as low, medium, high, many, few, tall, short, etc. deal with the approximate reasoning and data ambiguity concepts. These variables are described by specific type of functions depicting the relative graded membership of each linguistic term. The stated membership functions map each linguistic variable to a value between 0 and 1 , determining the degree of belongingness of a certain crisp system variable to the associated fuzzy linguistic variable on a steady scale from 0 to 1 . These variables take values from the fuzzy set specified by the range of points within the universe of discourse for the fuzzy variable. Typically, a fuzzy logic design architecture comprises of four main modules [18]:

- Fuzzy Rule Database: This module comprises of a set of fuzzy control rules for developing the control actions characterizing the dynamic system performance. These rules symbolized by the If-Then conditions describe the relationship between the crisp data and the corresponding fuzzy set model.

- Fuzzification Interface: The fuzzification interface transforms the crisp input data into grades of membership for the fuzzy linguistic variables.

- Fuzzy Inference Engine: The core component of a fuzzy logic controller is the fuzzy inference engine whose task is processing of data by means of mapping the input space into the output space through appropriate membership functions and set of fuzzy control rules.

- Defuzzification Interface: The defuzzification interface maps the fuzzy linguistic variables back into the space of corresponding numerical crisp values.

There are different ways to implement defuzzification process such as center of gravity, middle of maximum, weighted fuzzy mean, center of area, adaptive integration, bisector of area, etc. The supervised execution of the MIMO fuzzy system is used for controlling the decision-making systems under uncertainty. This is realized by the parallel implementation of rules induced via the proposed fuzzy heuristic approach.

\section{PROPOSED SYSTEM MODEL}

The basic architecture of the proposed fuzzy model for sustainable buildings design is demonstrated in Figure 1. This structured framework with heuristic fuzzy computing employs 
six input and four output attributes relevant to the optimal formulation of the green sustainable buildings. The first input of renewable energy consumption is focused on the utilization of renewable energy sources such as wind power generation, solar photovoltaic system, geothermal power, etc. to accomplish the sustainable development of buildings. Healthful interior environment of building standards associated with the occupants' comfort, hygiene, and minimal exposure to the pollutants is ensured by the second attribute of indoor air quality metric. With the increasing global demand for water, water use mitigation and rain water harvesting through water conservation technologies is apprehended as the third input to the presented fuzzy design model. Delivering services with the upgraded facilities to meet the ever changing provisions of clients is concretized through the fourth input parameter of services accessibility. The fifth input characterizes the incorporation of the recycled and alternative material content for the design and construction of green ecofriendly building architectures. In addition, combating the greenhouse gas emissions through the sustainable building development and operation implementations is the auxiliary setup goal. Furthermore, the fundamental performance indicators of energy efficiency, user satisfaction, resources optimization and environment quality correlated with the lifecycle and maintenance of sustainable buildings are envisaged as the four outputs of the fuzzy inference system. The considerable significance of these distinctive input and output attributes is contemplated by employing the MIMO fuzzy control discipline. The relationship between the multiple diverse input and output variables is illustrated in Table 1. For instance, the fuzzy output energy efficiency is determined by the input attributes of renewable energy consumption and recycled material content. Each of these inputs are characterized by the Low, Average and High fuzzy linguistic terms. Thus, the energy efficiency output requires a total of $\left(3^{2}\right)=9$ fuzzy rules. The second fuzzy output of user satisfaction is specified using the fuzzy input variables of indoor air quality, services accessibility, recycled material and greenhouse gas emissions identified with 2,2,3 and 2 linguistic variables. This contributes to 24 fuzzy rules. Likewise, the third fuzzy output resources optimization depends on the input variables of renewable energy consumption, water conservation and recycled material content, comprehensively requiring $\left(3^{3}\right)=27$ fuzzy rules. The fourth output variable of environment quality factor determined by the input parameters of renewable energy consumption, indoor air quality, recycled material, and greenhouse gas emissions, is designated by $(3 * 2 * 3 * 2)=36$ fuzzy rules. The commonly applied triangular, trapezoidal, Gaussian, Gaussian combination and generalized bell-shaped membership functions are utilized to represent these various fuzzy inputs and outputs as illustrated in Figures 2 and 3 . Here, the $x$-axis represents the designated range of the specific input/output dimension, and the y-axis denotes the corresponding degree of membership for that particular variable. Table 2 lists the different linguistic terms corresponding to the fuzzy inputs and outputs, together with their implemented membership functions and the associated fuzzy numbers. Mathematically, these characteristic functions are illustrated in the generalized Eqns. (1)-(4). Here, $\omega$ is the vector of numerical input values that are evaluated using the specific membership function. Triangular and trapezoidal fuzzy sets identified by linear membership functions and nonsmooth crossover points are defined using three $\left(x_{1}, x_{2}, x_{3}\right)$ and four $\left(x_{1}, x_{2}, x_{3}, x_{4}\right)$ parameters, respectively.
Also, the non-linear generalized bell-shaped membership function defined in Eq. (3) is described in terms of the width $x_{1}$, the center $x_{3}$, and the parameter $x_{2}$ regulating the slope of the membership curve. Similarly, the non-linear Gaussian (or Gaussian combination) membership function outlined in Eq. (4) is specified in terms of the center $x_{1}$, and the width $x_{2}$ of the membership curve. For both the generic bell-shaped and Gaussian membership functions, these various parameters determine the smoothness of the non-linear function around the transitions. Accordingly, the fuzzy rule base in our proposed optimization model comprises of the overall 96 inference rules as demonstrated in Table 3.

$$
\begin{gathered}
f\left(\omega ; x_{1}, x_{2}, x_{3}\right) \\
=\left\{\begin{array}{cc}
0 & \omega \leq x_{1} \\
\left(\omega-x_{1}\right) /\left(x_{2}-x_{1}\right) & x_{1} \leq \omega \leq x_{2} \\
\left(x_{3}-\omega\right) /\left(x_{3}-x_{2}\right) & x_{2} \leq \omega \leq x_{3} \\
0 & x_{3} \leq \omega
\end{array}\right\} \\
\left\{\begin{array}{cc}
0 & \omega \leq x_{1} \\
\left(\omega-x_{1}\right) /\left(x_{2}-x_{1}\right) & x_{1} \leq \omega \leq x_{2} \\
1 & x_{2} \leq \omega \leq x_{3} \\
\left(x_{4}-\omega\right) /\left(x_{4}-x_{3}\right) & x_{3} \leq \omega \leq x_{4} \\
0 & x_{4} \leq \omega
\end{array}\right\} \\
f\left(\omega ; x_{1}, x_{2}, x_{3}\right)=\frac{\left.x_{4}\right)=}{1+\left|\frac{\omega-x_{3}}{x_{1}}\right|^{2 x_{2}}} \\
f\left(\omega ; x_{1}, x_{2}\right)=e^{\frac{-\left(\omega-x_{1}\right)^{2}}{2 x_{2}^{2}}}
\end{gathered}
$$

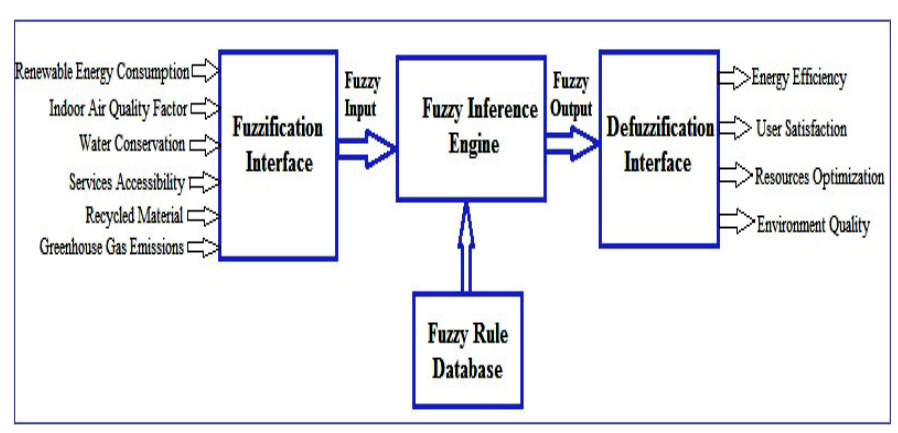

Figure 1. Basic structure of the proposed fuzzy based sustainable buildings model

Table 1. Relationship between input and output variables

\begin{tabular}{cc}
\hline Output Variable & Input Variable \\
\hline Energy Efficiency & Renewable Energy Consumption, \\
& Recycled Material \\
User Satisfaction & Indoor Air Quality Factor, \\
& Services Accessibility, \\
& Recycled Material, \\
& Greenhouse Gas Emissions \\
Resources Optimization & Renewable Energy Consumption, \\
& Water Conservation, \\
& Recycled Material \\
Environment Quality & Renewable Energy Consumption, \\
& Indoor Air Quality Factor, \\
& Recycled Material, \\
& Greenhouse Gas Emissions \\
\hline
\end{tabular}


Furthermore, our model uses the widely employed center of gravity or centroid method of defuzzification, in which the defuzzified value $\Psi$ is calculated as follows:

$$
\Psi=\frac{\int_{\Psi^{\min }}^{\Psi^{\max }} f(\Psi) * \Psi d \Psi}{\int_{\Psi^{\min }}^{\Psi^{\max }} f(\Psi) d \Psi}
$$

This defuzzified value is the abscissa of the center of gravity of the fuzzy set $f$ defined over the universe of discourse $\Psi$ of the output variables. Here, the denominator $\int_{\psi^{\min }}^{\Psi^{\max }} f(\Psi) d \Psi$ denotes the area of the region bounded by the membership curve over the interval $\left(\Psi^{\min }, \Psi^{\max }\right)$. The fuzzy inference method deployed in this analysis paradigm is the Mamdani method. This inference technique involves decision-making with respect to input categorization and concurrent intelligent computing. In the Mamdani inference system, the output variables are represented by fuzzy sets, which subsequently require defuzzification to convert these fuzzy output variables back into the space of corresponding crisp output variables.

Table 2. Fuzzy linguistic terms and their corresponding membership functions and fuzzy numbers for different input and output variables

\begin{tabular}{|c|c|c|c|}
\hline Variable & $\begin{array}{c}\text { Linguistic } \\
\text { Term }\end{array}$ & $\begin{array}{l}\text { Membership } \\
\text { Function }\end{array}$ & Fuzzy Numbers \\
\hline & Low & Trapezoidal & $\begin{array}{c}-18.12-5.8566 .111 \\
18.12]\end{array}$ \\
\hline $\begin{array}{l}\text { Renewable } \\
\text { Energy }\end{array}$ & Average & $\begin{array}{c}\text { Gaussian } \\
\text { Combination }\end{array}$ & {$\left[\begin{array}{llll}7.2 & 27.6 & 8.154 & 28.2\end{array}\right]$} \\
\hline Consumption & High & Trapezoidal & {$\left[\begin{array}{lllll}388.4 & 54.05 & 62.4 & 81.6\end{array}\right]$} \\
\hline $\begin{array}{l}\text { Indoor Air } \\
\text { Quality }\end{array}$ & Low & $\begin{array}{l}\text { Generalized } \\
\text { bell-shaped }\end{array}$ & {$\left[\begin{array}{lll}0.2 & 2.5 & 0.06614\end{array}\right]$} \\
\hline
\end{tabular}

\begin{tabular}{|c|c|c|c|}
\hline Factor & High & $\begin{array}{l}\text { Generalized } \\
\text { bell-shaped }\end{array}$ & [0.2 2.50 .9074$]$ \\
\hline $\begin{array}{c}\text { Water } \\
\text { Conservation }\end{array}$ & $\begin{array}{c}\text { Low } \\
\text { Average } \\
\text { High }\end{array}$ & $\begin{array}{l}\text { Trapezoidal } \\
\text { Triangular } \\
\text { Trapezoidal }\end{array}$ & $\begin{array}{c}{\left[\begin{array}{llll}-36 & -4 & 4 & 36\end{array}\right]} \\
{\left[\begin{array}{llll}20 & 50 & 80.03\end{array}\right]} \\
{\left[\begin{array}{llll}64 & 96 & 104 & 136\end{array}\right]}\end{array}$ \\
\hline $\begin{array}{c}\text { Services } \\
\text { Accessibility }\end{array}$ & $\begin{array}{l}\text { Low } \\
\text { High }\end{array}$ & $\begin{array}{l}\text { Trapezoidal } \\
\text { Trapezoidal }\end{array}$ & 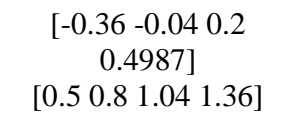 \\
\hline $\begin{array}{l}\text { Recycled } \\
\text { Material }\end{array}$ & $\begin{array}{c}\text { Low } \\
\text { Average } \\
\text { High }\end{array}$ & $\begin{array}{l}\text { Triangular } \\
\text { Trapezoidal } \\
\text { Triangular }\end{array}$ & $\begin{array}{c}{\left[\begin{array}{lll}-40 & 0 & 40\end{array}\right]} \\
{\left[\begin{array}{llll}20 & 46 & 54 & 80.03\end{array}\right]} \\
{\left[\begin{array}{llll}60 & 100 & 140\end{array}\right]}\end{array}$ \\
\hline $\begin{array}{l}\text { Greenhouse } \\
\text { Gas } \\
\text { Emissions }\end{array}$ & High & $\begin{array}{c}\text { Gaussian } \\
\text { Combination } \\
\text { Gaussian } \\
\text { Combination }\end{array}$ & $\begin{array}{c}{[1.11 \mathrm{e}+04-6117} \\
1.11 \mathrm{e}+041.056 \mathrm{e}+04] \\
{[95106.442 \mathrm{e}+04} \\
95107.002 \mathrm{e}+04]\end{array}$ \\
\hline $\begin{array}{l}\text { Energy } \\
\text { Efficiency } \\
\text { (Output) }\end{array}$ & $\begin{array}{l}\text { Low } \\
\text { Medium } \\
\text { High }\end{array}$ & $\begin{array}{l}\text { Trapezoidal } \\
\text { Gaussian } \\
\text { Trapezoidal }\end{array}$ & $\begin{array}{c}{\left[\begin{array}{llll}-36 & -4 & 4 & 36\end{array}\right]} \\
{\left[\begin{array}{llll}13.99 & 50\end{array}\right]} \\
{\left[\begin{array}{llll}6 & 96 & 104 & 136\end{array}\right]}\end{array}$ \\
\hline $\begin{array}{l}\text { User } \\
\text { Satisfaction } \\
\text { (Output) }\end{array}$ & $\begin{array}{l}\text { Low } \\
\text { Medium } \\
\text { High }\end{array}$ & $\begin{array}{l}\text { Triangular } \\
\text { Trapezoidal } \\
\text { Triangular }\end{array}$ & $\begin{array}{c}{\left[\begin{array}{lll}-40 & 0 & 40\end{array}\right]} \\
{\left[\begin{array}{llll}14 & 46 & 54 & 86\end{array}\right]} \\
{\left[\begin{array}{llll}60 & 100 & 140\end{array}\right]}\end{array}$ \\
\hline $\begin{array}{l}\text { Resources } \\
\text { Optimization } \\
\text { (Output) }\end{array}$ & $\begin{array}{c}\text { Low } \\
\text { Medium } \\
\text { High }\end{array}$ & $\begin{array}{c}\text { Generalized } \\
\text { bell-shaped } \\
\text { Gaussian } \\
\text { Generalized } \\
\text { bell-shaped }\end{array}$ & 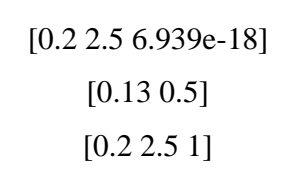 \\
\hline $\begin{array}{c}\text { Environment } \\
\text { Quality } \\
\text { (Output) }\end{array}$ & $\begin{array}{l}\text { Low } \\
\text { Medium } \\
\text { High }\end{array}$ & $\begin{array}{l}\text { Triangular } \\
\text { Triangular } \\
\text { Triangular }\end{array}$ & $\begin{array}{c}{\left[\begin{array}{lll}-0.4 & 0 & 0.4\end{array}\right]} \\
{\left[\begin{array}{lll}0.1 & 0.5 & 0.9\end{array}\right]} \\
{\left[\begin{array}{lll}0.6 & 1 & 1.4\end{array}\right]}\end{array}$ \\
\hline
\end{tabular}

Table 3. Fuzzy inference rules for the proposed fuzzy based sustainable building model 


\begin{tabular}{|c|c|c|c|c|c|c|c|c|c|c|}
\hline S. \# & REC & IAQF & WC & $\mathbf{S A}$ & $\mathbf{R M}$ & GGE & $\mathbf{E E}$ & US & RO & EQ \\
\hline 29 & - & High & - & High & Low & High & - & Med & - & - \\
\hline 30 & - & High & - & High & Avg & Low & - & High & - & - \\
\hline 31 & - & High & - & High & Avg & High & - & Med & - & - \\
\hline 32 & - & High & - & High & High & Low & - & High & - & - \\
\hline 33 & - & High & - & High & High & High & - & Med & - & - \\
\hline 34 & Low & - & Low & - & Low & - & - & - & Low & - \\
\hline 35 & Low & - & Low & - & Avg & - & - & - & Med & - \\
\hline 36 & Low & - & Low & - & High & - & - & - & Med & - \\
\hline 37 & Low & - & Avg & - & Low & - & - & - & Low & - \\
\hline 38 & Low & - & Avg & - & Avg & - & - & - & Med & - \\
\hline 39 & Low & - & Avg & - & High & - & - & - & Med & - \\
\hline 40 & Low & - & High & - & Low & - & - & - & Med & - \\
\hline 41 & Low & - & High & - & Avg & - & - & - & High & - \\
\hline 42 & Low & - & High & - & High & - & - & - & High & - \\
\hline 43 & Avg & - & Low & - & Low & - & - & - & Low & - \\
\hline 44 & Avg & - & Low & - & Avg & - & - & - & Low & - \\
\hline 45 & Avg & - & Low & - & High & - & - & - & Med & - \\
\hline 46 & Avg & - & Avg & - & Low & - & - & - & Low & - \\
\hline 47 & Avg & - & Avg & - & Avg & - & - & - & Med & - \\
\hline 48 & Avg & - & Avg & - & High & - & - & - & Med & - \\
\hline 49 & Avg & - & High & - & Low & - & - & - & Med & - \\
\hline 50 & Avg & - & High & - & Avg & - & - & - & High & - \\
\hline 51 & Avg & - & High & - & High & - & - & - & High & - \\
\hline 52 & High & - & Low & - & Low & - & - & - & Low & - \\
\hline 53 & High & - & Low & - & Avg & - & - & - & Low & - \\
\hline 54 & High & - & Low & - & High & - & - & - & Med & - \\
\hline 55 & High & - & Avg & - & Low & - & - & - & Low & - \\
\hline 56 & High & - & Avg & - & Avg & - & - & - & Low & - \\
\hline 57 & High & - & Avg & - & High & - & - & - & Med & - \\
\hline 58 & High & - & High & - & Low & - & - & - & Med & - \\
\hline 59 & High & - & High & - & Avg & - & - & - & Med & - \\
\hline 60 & High & - & High & - & High & - & - & - & Med & - \\
\hline 61 & Low & Low & - & - & Low & Low & - & - & - & Low \\
\hline 62 & Low & Low & - & - & Low & High & - & - & - & Low \\
\hline 63 & Low & Low & - & - & Avg & Low & - & - & - & Med \\
\hline 64 & Low & Low & - & - & Avg & High & - & - & - & Low \\
\hline 65 & Low & Low & - & - & High & Low & - & - & - & Med \\
\hline 66 & Low & Low & - & - & High & High & - & - & - & Low \\
\hline 67 & Low & High & - & - & Low & Low & - & - & - & High \\
\hline 68 & Low & High & - & - & Low & High & - & - & - & Med \\
\hline 69 & Low & High & - & - & Avg & Low & - & - & - & Med \\
\hline 70 & Low & High & - & - & Avg & High & - & - & - & Med \\
\hline 71 & Low & High & - & - & High & Low & - & - & - & High \\
\hline 72 & Low & High & - & - & High & High & - & - & - & Med \\
\hline 73 & Avg & Low & - & - & Low & Low & - & - & - & Low \\
\hline 74 & Avg & Low & - & - & Low & High & - & - & - & Low \\
\hline 75 & Avg & Low & - & - & Avg & Low & - & - & - & Low \\
\hline 76 & Avg & Low & - & - & Avg & High & - & - & - & Low \\
\hline 77 & Avg & Low & - & - & High & Low & - & - & - & Low \\
\hline 78 & Avg & Low & - & - & High & High & - & - & - & Low \\
\hline 79 & Avg & High & - & - & Low & Low & - & - & - & Med \\
\hline 80 & Avg & High & - & - & Low & High & - & - & - & Med \\
\hline 81 & Avg & High & - & - & Avg & Low & - & - & - & Med \\
\hline 82 & Avg & High & - & - & Avg & High & - & - & - & Med \\
\hline 83 & Avg & High & - & - & High & Low & - & - & - & High \\
\hline 84 & Avg & High & - & - & High & High & - & - & - & Med \\
\hline 85 & High & Low & - & - & Low & Low & - & - & - & Low \\
\hline 86 & High & Low & - & - & Low & High & - & - & - & Low \\
\hline 87 & High & Low & - & - & Avg & Low & - & - & - & Low \\
\hline 88 & High & Low & - & - & Avg & High & - & - & - & Low \\
\hline 89 & High & Low & - & - & High & Low & - & - & - & Low \\
\hline 90 & High & Low & - & - & High & High & - & - & - & Low \\
\hline 91 & High & High & - & - & Low & Low & - & - & - & Med \\
\hline 92 & High & High & - & - & Low & High & - & - & - & Med \\
\hline 93 & High & High & - & - & Avg & Low & - & - & - & Med \\
\hline 94 & High & High & - & - & Avg & High & - & - & - & Low \\
\hline 95 & High & High & - & - & High & Low & - & - & - & High \\
\hline 96 & High & High & - & - & High & High & - & - & - & Low \\
\hline
\end{tabular}



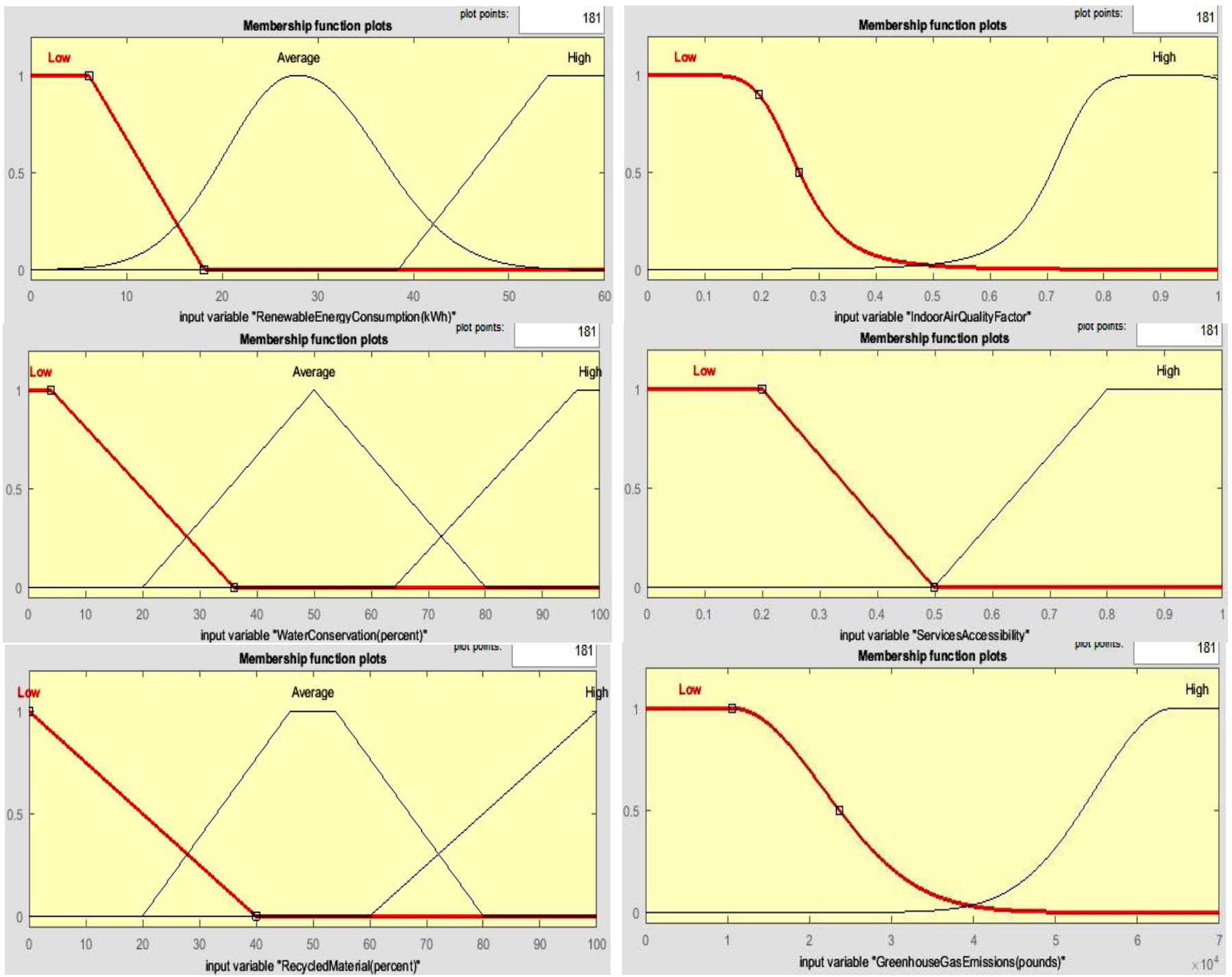

Figure 2. Fuzzy input membership functions
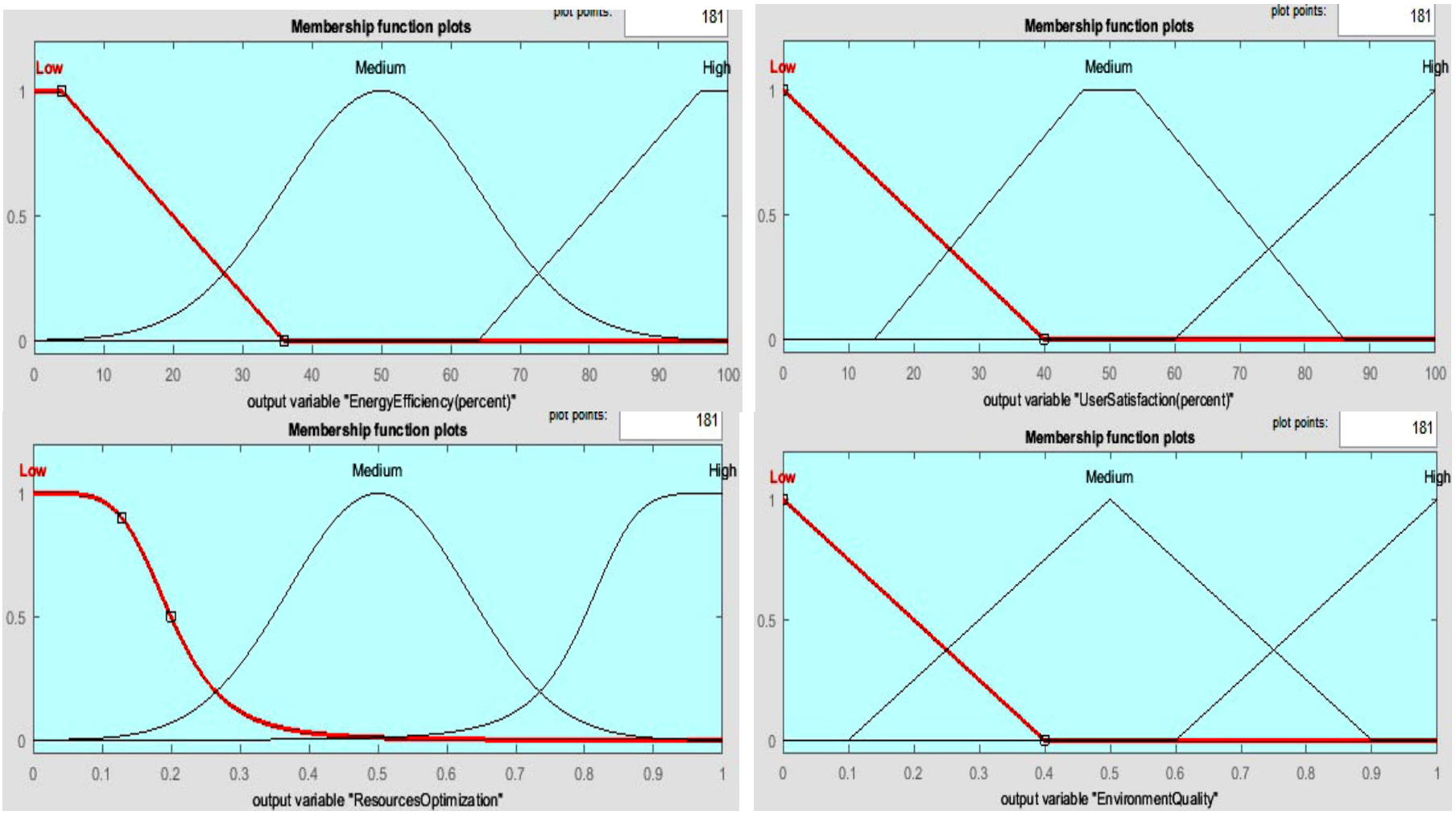

Figure 3. Fuzzy output membership functions 


\section{SIMULATION RESULTS AND ANALYSIS}

The simulation of the proposed sustainability model is executed in the Matlab software [19]. Particularly, we employ the Fuzzy Logic Toolbox Graphical User Interface tool to design and implement the developed green building system. In Figure 4, we plot the rule viewer structure such that given the inputs as: Renewable Energy Consumption $=16 \mathrm{kWh}$, Indoor Air Quality Factor $=0.713$, Water Conservation $=75.5 \%$, Services Accessibility $=0.585$, Recycled Material $=64.9 \%$, Greenhouse Gas Emissions $=24,600$ pounds, then the corresponding outputs Energy Efficiency $=54.6 \%$, User Satisfaction $=82.4 \%$, Resources Optimization $=0.654$, and Environment Quality $=0.534$, that are indicative of Medium Energy Efficiency, High User Satisfaction, Medium Resources Optimization, and Medium Environment Quality for the specified inputs. Figure 5 illustrates the threedimensional response surfaces exhibiting the fuzzy system outputs for various combinations of input variables. For each fuzzy output attribute, we plot the decision curve as a function of the two most significant fuzzy inputs determining the evolution of the system output.

The presented fuzzy modeling scheme is trained with a sample dataset corresponding to the sustainable building design framework. The model is verified by varying the size of data collection from 1 to 30 . Table 4 depicts the values of different inputs for this dataset concatenated with the values of the outputs computed using the conventional method and the outputs estimated by the proposed fuzzy logic design analysis. This sample data collection comprising of the synthetic data is used to generate a model for improving the system performance on the subsequent unseen data.

Figure 6 illustrates the actual and the predicted values of energy efficiency metric for each dataset instance. On an average, energy efficiency computed using the proposed fuzzy model is $7.85 \%$ higher than the standard modeling technique. The observed and the corresponding estimated values of user satisfaction output are plotted in Figure 7. It can be assessed that the proposed optimization model demonstrates $14.34 \%$ higher accuracy in estimating the occupants' satisfaction and comfort. In a similar manner, Figures 8 and 9 represent the actual and predicted values of resources optimization and environment quality output variables, respectively. Our model exhibits the improved resource utilization and enhanced environment protection relatively by a factor of 1.0586 and 1.0594 as compared with the conventional method.

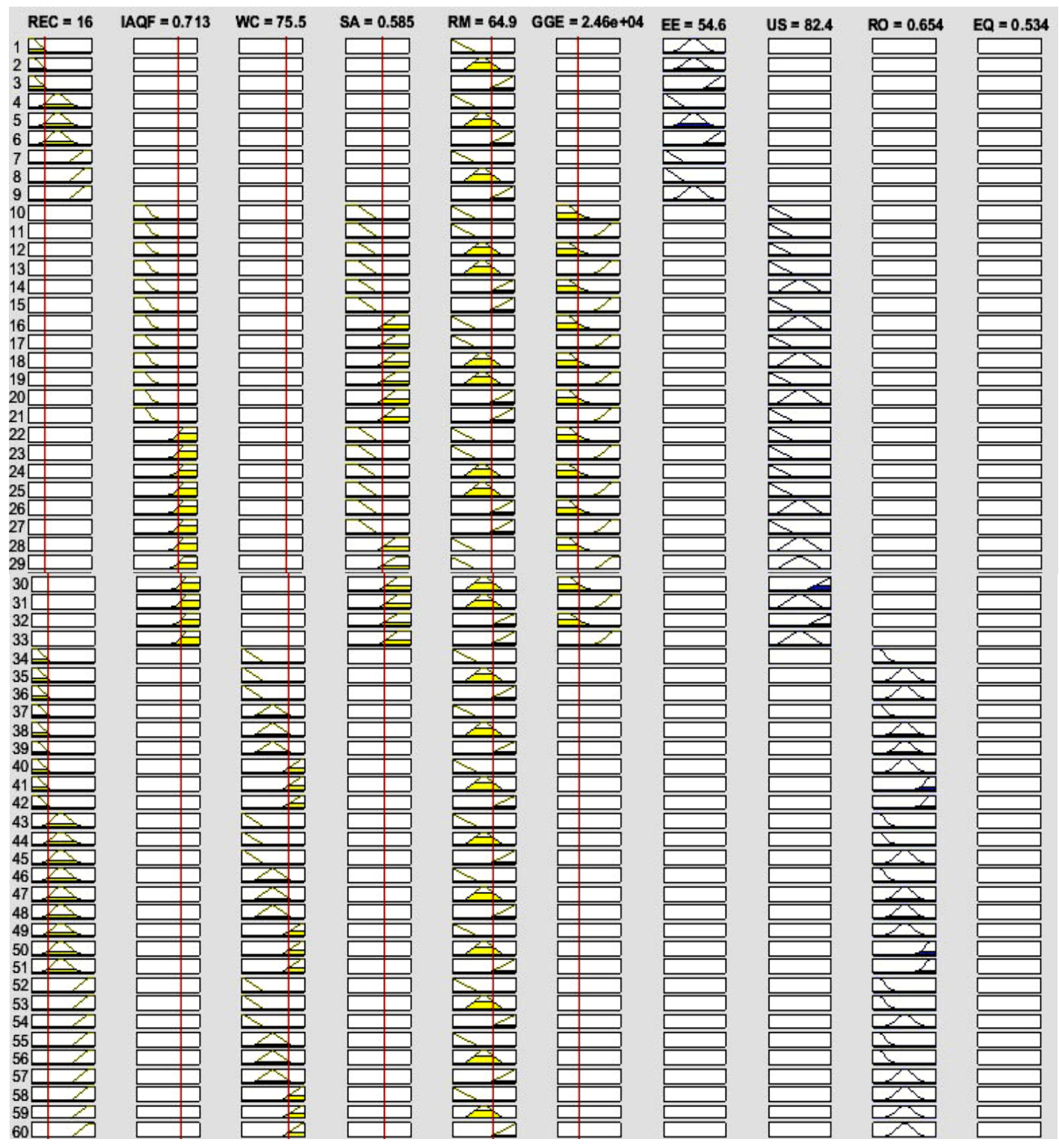



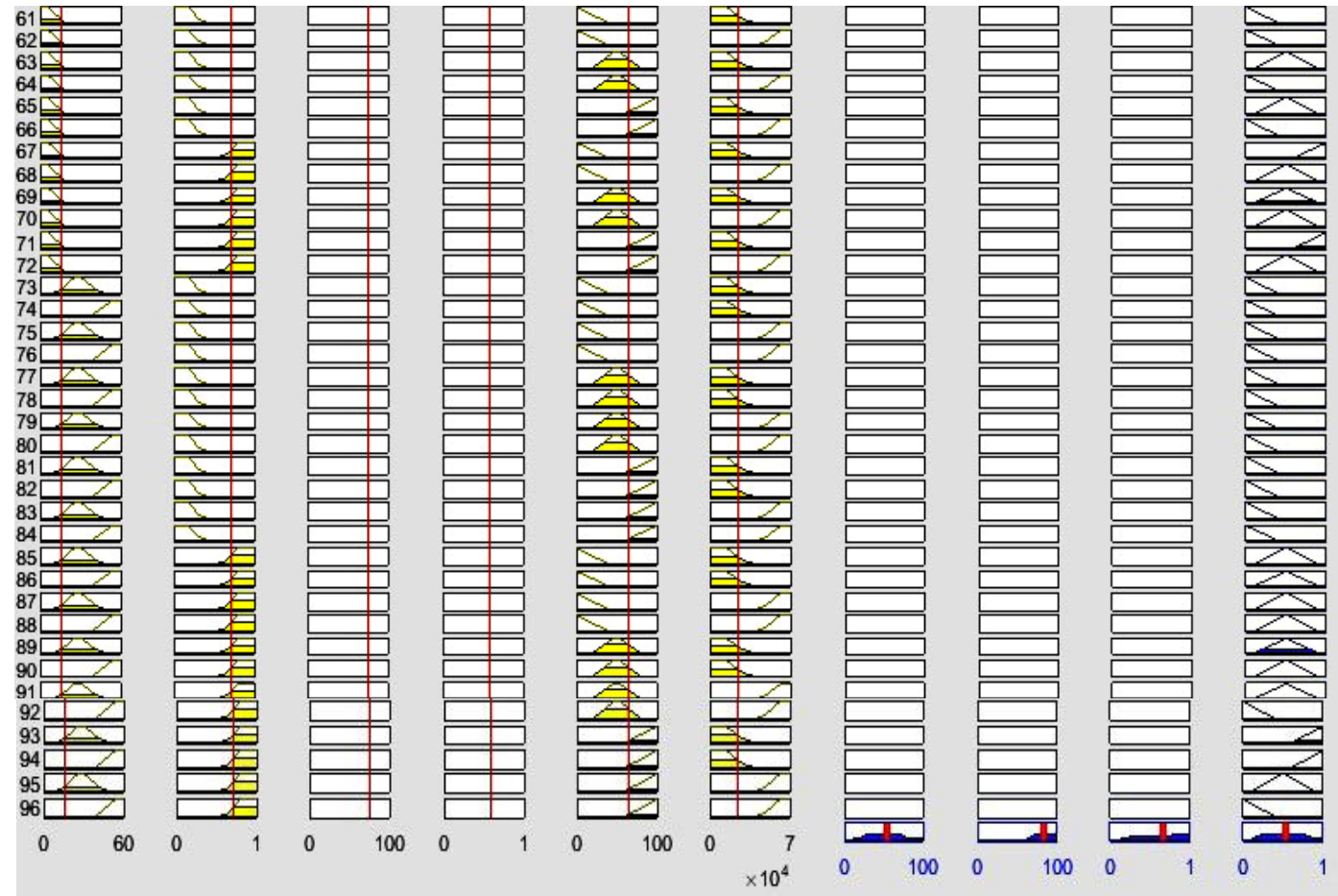

Figure 4. Rule viewer of the proposed fuzzy model


Figure 5. Three-dimensional decision surface viewer

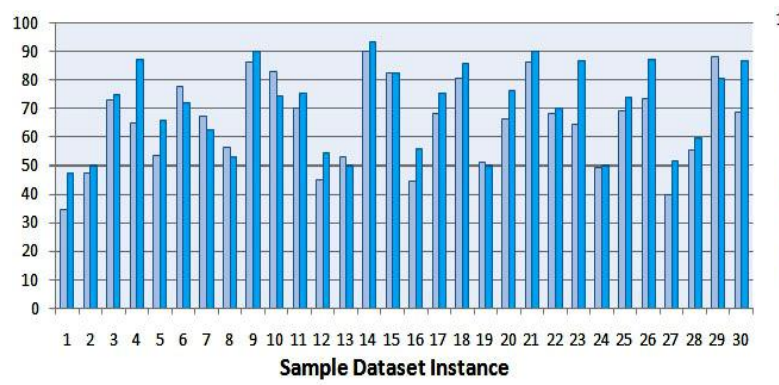

Sample Dataset Instance

$\square$ Actual Energy Efficiency $(\%) \quad \square$ Predicted Energy Efficiency $(\%)$

Figure 6. Actual and observed values of energy efficiency metric for each dataset instance

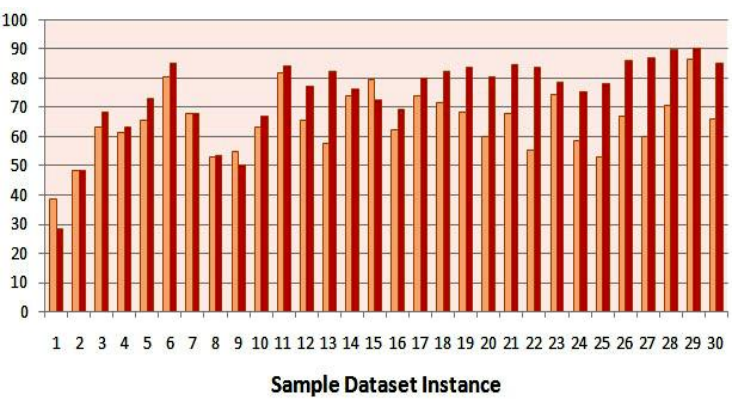

$\square$ Actual User Satisfaction (\%) $\quad$ Predicted User Satisfaction (\%)

Figure 7. Actual and observed values of user satisfaction metric for each dataset instance 
Table 4. Sample dataset for the proposed fuzzy-based sustainable building model

\begin{tabular}{|c|c|c|c|c|c|c|c|c|c|c|c|c|c|c|}
\hline $\begin{array}{l}\text { S. } \\
\text { No. }\end{array}$ & REC & IAQF & WC & SA & RM & GGE & $\begin{array}{l}\text { Actua } \\
\text { l EE }\end{array}$ & $\begin{array}{c}\text { Predicte } \\
\text { d EE }\end{array}$ & $\begin{array}{l}\text { Actua } \\
\text { I US }\end{array}$ & $\begin{array}{c}\text { Predicte } \\
\text { d US }\end{array}$ & $\begin{array}{c}\text { Actual } \\
\text { RO }\end{array}$ & $\begin{array}{c}\text { Predicte } \\
\text { d RO }\end{array}$ & $\begin{array}{c}\text { Actual } \\
\text { EQ }\end{array}$ & $\begin{array}{c}\text { Predicte } \\
\text { d EQ }\end{array}$ \\
\hline 1 & 20 & 0.35 & 40 & 0.2 & 36 & 45000 & & 47.8 & 38.82 & 28.5 & 0.364 & 0.473 & 0.421 & 0.212 \\
\hline 2 & 32 & 0.62 & 65 & 0.45 & 42 & 32000 & 47.66 & 50.0 & 48.6 & 48.4 & 0.534 & 0.507 & 0.507 & 0.497 \\
\hline 3 & 47 & 0.55 & 54 & 0.6 & 68 & 48500 & 73.16 & 74.7 & 63.1 & 68.5 & 0.667 & 0.7 & 0.676 & 0.75 \\
\hline 4 & 25 & 0.4 & 66 & 0.8 & 88 & 26000 & 64.83 & 87.2 & 61.3 & 63.1 & 0.652 & 0.67 & 0.517 & 0.664 \\
\hline 5 & 19 & 0.68 & 75 & 0.69 & 75 & 36000 & 53.33 & 65.8 & 65.8 & 73.2 & 0.605 & 0.669 & 0.565 & 0.6 \\
\hline 6 & 41.5 & 0.72 & 80 & 0.86 & 86 & 55000 & 77.58 & 72.1 & 80.6 & 85.3 & 0.783 & 0.74 & 0.764 & 0.72 \\
\hline 7 & 36.8 & 0.9 & 72.5 & 0.55 & 73.2 & 37500 & 67.26 & 62.4 & 67.9 & 68.1 & 0.69 & 0.616 & 0.695 & 0.77 \\
\hline 8 & 28.5 & 0.45 & 46.5 & 0.67 & 65.5 & 24000 & 56.5 & 53.3 & 52.9 & 53.5 & 0.532 & 0.5 & 0.48 & 0.8 \\
\hline 9 & 51 & 0.8 & 92.2 & 0.36 & 87.5 & 11000 & 86.25 & 90.2 & 54.8 & 50.2 & 0.882 & 0.81 & 0.67 & 0.857 \\
\hline 10 & 44.2 & 0.71 & 83.7 & 0.36 & 92 & 37680 & & 74.5 & 63.2 & 66.8 & 0.831 & 0.85 & 0.726 & 0.77 \\
\hline 11 & 37.5 & 0.85 & 58.4 & 0.75 & 78.2 & 62500 & 70.35 & 75.4 & 81.8 & 84.2 & 0.663 & 0.9 & 0.787 & 0.7 \\
\hline 12 & 15.8 & 0.94 & 45.5 & 0.42 & 64.5 & 42750 & 45.41 & 54.3 & 65.4 & 77.3 & 0.454 & 0.91 & 0.614 & 0.67 \\
\hline 13 & 31.4 & 0.78 & 66.5 & 0.59 & 53.4 & 28500 & 52.86 & 50.1 & 57.7 & 82.4 & 0.574 & 0.8 & 0.561 & 0.7 \\
\hline 14 & 58.5 & 0.52 & 61.9 & 0.78 & 82.5 & 58500 & 90.0 & 93.3 & 74.1 & 76.2 & 0.806 & 0.83 & 0.788 & 0.71 \\
\hline 15 & 45.4 & 0.83 & 88.5 & 0.93 & 89.6 & 36410 & 82.63 & 82.3 & 79.4 & 72.4 & 0.845 & 0.8 & 0.75 & 0.69 \\
\hline 16 & 12.5 & 0.9 & 74.1 & 0.25 & 68.5 & 46750 & 44.66 & 56.1 & 62.5 & 69.5 & 0.544 & 0.648 & 0.615 & 0.502 \\
\hline 17 & 35 & 0.75 & 77.5 & 0.68 & 78.2 & 52500 & 68.26 & 75.4 & 74.0 & 80.1 & 0.713 & 0.743 & 0.716 & 0.52 \\
\hline 18 & 38 & 0.85 & 52.6 & 0.39 & 98 & 44780 & 80.66 & 85.8 & 71.5 & 82.1 & 0.713 & 0.5 & 0.775 & 0.71 \\
\hline 19 & 27.8 & 0.93 & 65 & 0.875 & 56.2 & 25600 & & 50.0 & 68.3 & 83.8 & 0.558 & 0.6 & 0.58 & 0.72 \\
\hline 20 & 41.15 & 0.64 & 58.5 & 0.725 & 63.7 & 28000 & 66.14 & 76.5 & 60.0 & 80.5 & 0.635 & 0.67 & 0.59 & 0.8 \\
\hline 21 & 54.5 & 0.8 & 96 & 0.88 & 81.5 & 15500 & 86.16 & 90.5 & 67.9 & 84.8 & 0.894 & 0.81 & 0.686 & 0.847 \\
\hline 22 & 46.38 & 0.7 & 85.2 & 0.65 & 58.9 & 19850 & 68.1 & 70.2 & 55.5 & 83.9 & 0.738 & 0.7 & 0.586 & 0.499 \\
\hline 23 & 25.8 & 0.75 & 72.8 & 0.9 & 85.66 & 33500 & 64.33 & 86.7 & 74.6 & 78.8 & 0.671 & 0.621 & 0.628 & 0.786 \\
\hline 24 & 30.54 & 0.58 & 33.45 & 0.86 & 48.5 & 29880 & 49.7 & 50.5 & 58.7 & 75.5 & 0.442 & 0.476 & 0.5 & 0.53 \\
\hline 25 & 42.9 & 0.68 & 74.8 & 0.265 & 66.6 & 36350 & 69.05 & 74.3 & 53.2 & 78.0 & 0.71 & 0.65 & 0.645 & 0.7 \\
\hline 26 & 34.7 & 0.87 & 65.2 & 0.78 & 89.0 & 10500 & 73.41 & 87.2 & 67.2 & 86.0 & 0.706 & 0.74 & 0.622 & 0.86 \\
\hline 27 & 9.88 & 0.46 & 22.5 & 0.96 & 63.35 & 24780 & 39.91 & 51.7 & 60.1 & 87.2 & 0.341 & 0.486 & 0.403 & 0.475 \\
\hline 28 & 23.6 & 0.69 & 89.6 & 0.62 & 71.4 & 56190 & & 60.3 & 70.6 & 89.8 & 0.667 & 0.841 & 0.649 & 0.499 \\
\hline 29 & 48.5 & 0.85 & 98.8 & 0.97 & 95.7 & 48300 & 88.26 & 80.7 & 86.6 & 90.1 & 0.917 & 0.87 & 0.826 & 0.675 \\
\hline 30 & 32.24 & 0.742 & 79.5 & 0.75 & 84.15 & 21500 & 68.94 & 86.5 & 66.0 & 85.1 & 0.724 & 0.89 & 0.606 & 0.851 \\
\hline
\end{tabular}

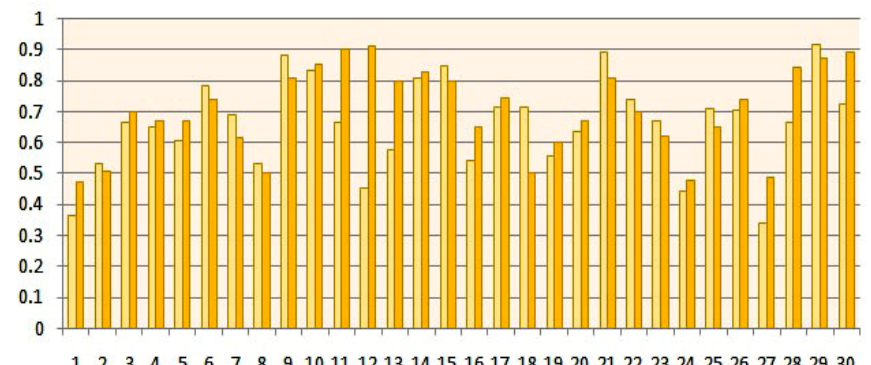

Sample Dataset Instance

$\square$ Actual Resources Optimization $\quad \square$ Predicted Resources Optimization

Figure 8. Actual and observed values of resources optimization metric for each dataset instance



Figure 9. Actual and observed values of environment quality metric for each dataset instance

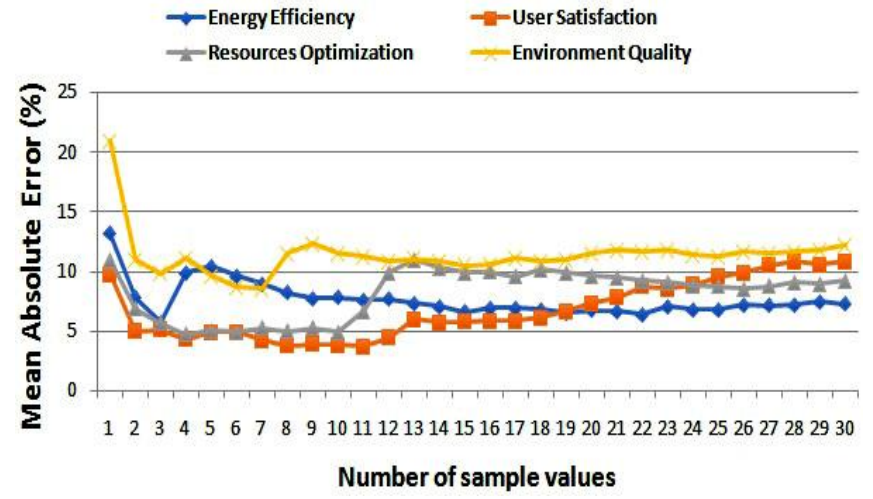

Figure 10. Mean absolute error of the four fuzzy outputs plotted as a function of varying number of sample values

Mean absolute error estimation is a Bayesian approach to measure the performance of random fuzzy output metrics. This error measurement for the contemplated four fuzzy outputs is depicted graphically in Figure 10 with the evolving number of sample dataset values. It can be evaluated that these output parameters present $7.6 \%, 6.74 \%, 8.13 \%$, and $11.3 \%$ mean absolute error in estimating their optimal solutions through the proposed model design. Analogously, Figure 11 shows the logarithmic quotient error measured during the computation of the explored output attributes for performance assessment of the sample data with the developed system model. This error is computed as $0.1303,0.12,0.1347$, and 0.221 for the corresponding performance metrics associated with globally sustainable fuzzy logic model. Therefore, the environment quality output exhibits maximal error and the user satisfaction 
output shows minimal error in estimating their optimal values through the proposed fuzzy design model of green buildings architecture.



Figure 11. Logarithmic quotient error of the four fuzzy outputs plotted as a function of varying number of sample values

\section{CONCLUSION}

In this work, an adaptive fuzzy design model is developed for multidimensional performance analysis of sustainable green buildings. This model contemplated several attributes influencing the efficient operation of these buildings with minimal environmental degradation and maximal user's satisfaction and comfort. The developed architecture for sustainable buildings is aimed at achieving optimal utilization of restrained resources and enhancing the energy efficacy during the set up and maintenance of resilient buildings. A sample data collection comprising of synthetic data about the specific operating sustainable site is used to generate a model assessment tool. This can be used for improving the proposed system performance on the subsequent unseen data of future building design model in its preliminary stages. The accuracy of the proposed model is measured in terms of various conventional error metrics including mean absolute error and logarithmic quotient error computed for the four output variables. It can be deduced from the simulation analysis results that both the metrics provide optimal solutions with up to $12 \%$ and $22 \%$ error approximations. This multivariate fuzzy logic model for design and analysis of sustainable buildings can provide architectural guidance to the future decisionmakers for optimal development of environmental-friendly and energy effective system formulation. Moreover, it minimizes the negative impact on global environment, toxic emissions, material costs, and scarce energy resources, while promoting the quality of services for residents and visitants. Compared with the traditional methods, the application of the computational intelligent fuzzy algorithm seems to be most efficient with minimal implementation complexity for multidimensional sustainability optimization in green building design. This model essentially accomplishes the diverse system objectives including the energy conservation, user convenience, resource utilization and environment protection through rigorous simulation for the experimental estimation and analysis.

The size of the considered sample dataset can be expanded in future to obtain simulation results with improved statistical efficiency and higher model accuracy. The interaction of social, technological and economic factors influencing the individual behaviour, and cost-effective evaluation and benefit analysis over a fixed period of time can be investigated for improved sustainability in building development. In addition, future directions may consider automated rule generation and optimization for scalable real-world datasets by employing advanced machine learning techniques. Further, it is suggested to utilize the evolutionary heuristic approaches such as genetic optimization, particle swarm optimization, etc. for implementing the multiple-attribute decision-making sustainability model to explore the efficacy and general applicability of the proposed technique.

\section{REFERENCES}

[1] Yao, J. (2014). A multi-objective (energy, economic and environmental performance) life cycle analysis for better building design. Sustainability, 6(2): 602-614. https://doi.org/10.3390/su6020602

[2] Ahn, Y.H., Jung, C.W., Suh, M., Jeon, M.H. (2016). Integrated construction process for green building. Procedia Engineering, 145: 670-676. https://doi.org/10.1016/j.proeng.2016.04.065

[3] Abualrejal, H.M., Udin, Z.M., Mohtar, S. (2017). Green building toward construction sustainability: Energy efficiency with material and design aspects. Journal of Technology and Operations Management, 12: 100-109.

[4] Ho, K.K.W., So, S. (2017). Towards a smart city through household recycling and waste management: A study on the factors affecting environmental friendliness lifestyle of Guamanian. International Journal of Sustainable Real Estate and Construction Economics, 1(1): 89-108. https://doi.org/10.1504/IJSRECE.2017.084385

[5] Singh, C.S. (2018). Green construction: Analysis on green and sustainable building techniques. Civil Engineering Research Journal, 4(3): 107-112. https://doi.org/10.19080/CERJ.2018.04.555638

[6] Blanco, I., Schettini, E., Vox, G. (2018). Effects of vertical green technology on building surface temperature. International Journal of Design \& Nature and Ecodynamics, 13(4): 384-394. https://doi.org/10.2495/DNE-V13-N4-384-394

[7] Oberti, I., Plantamura, F. (2017). The inclusion of natural elements in building design: The role of green rating systems. International Journal of Sustainable Development and Planning, 12(2): 217-226. https://doi.org/10.2495/SDP-V12-N2-217-226

[8] Patnaik, A., Kumar, V., Saha, P. (2018). Importance of indoor environmental quality in green buildings. Environmental Pollution, Water Science and Technology Library, 77: 53-64. https://doi.org/10.1007/978-981-105792-2_5

[9] Li, W., Wang, D.L., Qu, C.F., Zhang, X., Wu, W.J., Zhao, P. (2019). Energy consumption data analysis and operation evaluation of green buildings. Smart Innovations in Communication and Computational Sciences, Advances in Intelligent Systems and Computing, 851: 137-146. https://doi.org/10.1007/978981-13-2414-7_14

[10] Martins, A.I., Lima A.C.O., Bartolomeu, P., Mouzinho, L.F., Ferreira, J., Rocha, N.P. (2020). Enabling green building's comfort using information and 
communication technologies: A systematic review of the literature. Trends and Innovations in Information Systems and Technologies: WorldCIST 2020, Advances in Intelligent Systems and Computing, 1160: 197-208. https://doi.org/10.1007/978-3-030-45691-7_19

[11] Bhatt, A., Desai, S., Gulabani, S. (2019). Energyefficient green building with sustainable engineering of natural resources. Sustainable Engineering, Lecture Notes in Civil Engineering, 30: 329-336. https://doi.org/10.1007/978-981-13-6717-5_32

[12] Singh, R.V., Singh, K., Vyas, R. (2019). "Green Building": A step towards environmental and economic construction. International Journal of Environment and Climate Change, 9(7): 391-401. https://doi.org/10.9734/ijecc/2019/v9i730124

[13] Borissova, D. (2020). A multi-criteria group decision making model for selection of green building project. Advances in Building Information Modeling: EBF 2019, Communications in Computer and Information Science, 1188: 137-146. https://doi.org/10.1007/978-3-03042852-5_11

[14] Rajagopal, M., Karuppiah, G. (2019). Green environmental sustainability development in construction industry using response surface methodology. International Journal of Environment and Sustainable Development, 18(1): 13-44. https://doi.org/10.1504/IJESD.2019.098639

[15] Petrova, E., Pauwels, P., Svidt, K., Jensen, R.L. (2019). In search of sustainable design patterns: Combining data mining and semantic data modelling on disparate building data. Advances in Informatics and Computing in Civil and Construction Engineering, Springer, Cham, 19-26. https://doi.org/10.1007/978-3-030-00220-6_3

[16] Saleh, R.M., Anuar, M.M., Al-Swidi, A.K., Omar, K. (2020). The effect of awareness, knowledge and cost on intention to adopt green building practices. International Journal of Environment and Sustainable Development, 19(1): 33-58. https://doi.org/10.1504/IJESD.2020.105468

[17] Zadeh, L.A. (1965) 'Fuzzy sets. Information and Control, 8(3): $\quad 338-353 . \quad$ https://doi.org/10.1016/S00199958(65)90241-X

[18] Zimmerman, H.J. (2001). Fuzzy Set Theory and Its Applications. Fourth edition, Kluwer Academic Publishers Group.

[19] MATLAB

[Online]. http://www.mathworks.com/products/matlab/descriptio n1.html. 